\title{
DE LA ARQUITECTURA COLONIAL A LA ARQUITECTURA CONTEMPORÁNEA: OTRA MIRADA SOBRE LA FIGURA DE MARIO BUSCHIAZZO
}

Johanna Natalí Zimmerman

\section{MARIO J. BUSCHIAZZO}

DE LA CABAÑA AL RASCACIELOS

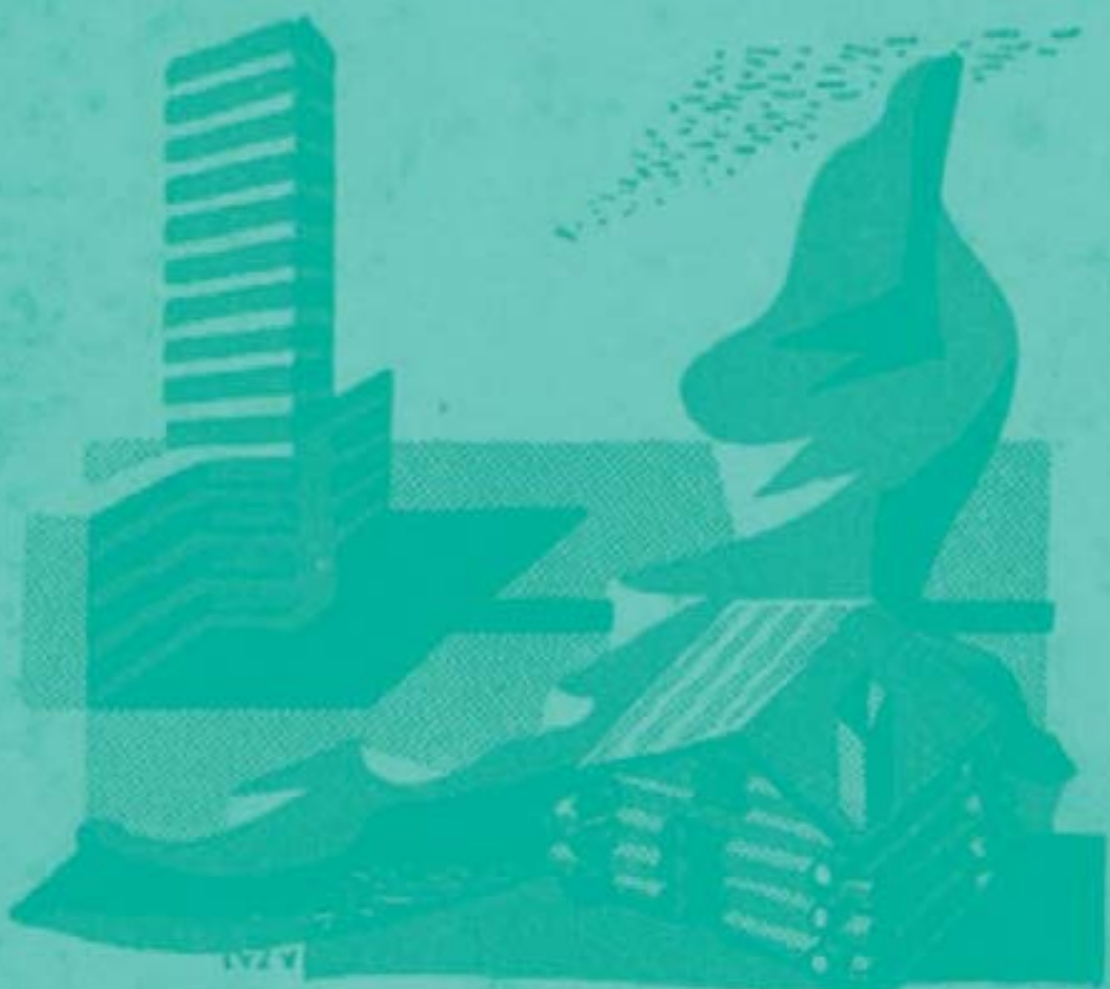




\section{JOHANNA NATALÍ ZIMMERMAN}

Arquitecta. Se encuentra terminando la Maestría en Historia y Cultura de la Arquitectura y la Ciudad en UTDT y cursando sus estudios de doctorado en FADU - UBA, con una beca doctoral UBACyT. Docente de Historia de la Arquitectura (cátedra Sabugo, FADU - UBA). 


\section{RESUMEN}

Mario José Buschiazzo suele ser reconocido en la literatura especializada por su aporte al conocimiento de la historia de la arquitectura, no solo argentina, sino también latinoamericana. Su trabajo como restaurador y, posteriormente, como investigador y motor de estudios relacionados con la historia de la arquitectura americana -sobre todo colonial- ha dejado una huella en el campo historiográfico. Hoy en día, Buschiazzo es un referente imprescindible a la hora de pensar la arquitectura colonial.

Sin embargo, su trayectoria deja entrever un perfil que poco parece tener que ver con su interés por lo colonial: su relación con la arquitectura americana contemporánea. Y esta relación se construye a partir de múltiples frentes que se relacionan, en gran parte, con la arquitectura de los Estados Unidos. A lo largo de esta investigación se estudiará la relación de Buschiazzo con la arquitectura americana contemporánea, principalmente entre los años 1955 (cuando el Instituto de Arte Americano e Investigaciones Estéticas lanza la colección "Arquitectos Americanos Contemporáneos") y 1970 (año de su fallecimiento), con el objetivo de construir una imagen más rica y compleja del arquitecto que aquella obtenida únicamente a partir de sus estudios acerca de lo colonial o de su trabajo como restaurador.

Palabras Clave: Mario Buschiazzo - Historiografía de la arquitectura en Argentina - Arquitectura argentina - Arquitectura Americana Contemporánea - Instituto de Arte Americano e Investigaciones Estéticas - Arquitectura colonial.

\section{ABSTRACT}

Mario José Buschiazzo is usually recognized in the literature for his contribution not only to Argentinean, but also to Latin-American history of architecture. His work as an architectural restorer and, later, as a researcher and promoter of studies related to the history of American architecture -mainly colonial architecture- has left a mark in the historiographical field. Nowadays, Buschiazzo is an essential reference when thinking about colonial architecture. However, his trajectory suggests a profile that has little in common with his interests for colonial architecture: his relationship with contemporary American architecture. This relationship involves multiple approaches that are largely related with architecture in the United States. This article studies Buschiazzo's relationship with contemporary American architecture, mainly between 1955 (when the Instituto de Arte Americano e Investigaciones Estéticas launches the "Arquitectos Americanos Contemporáneos" collection) and 1970 (the year of his death), with the objective of constructing a richer and more complex image of the architect than that obtained only through his studies about colonial architecture or his work as an architectural restorer.

Keywords: Mario Buschiazzo - Architectural historiography in Argentina - Argentinean Architecture - Contemporary American architecture - Instituto de Arte Americano e Investigaciones Estéticas - Colonial architecture. 


\section{DE LA ARQUITECTURA COLONIAL A LA ARQUITECTURA CONTEMPORÁNEA: OTRA MIRADA SOBRE LA FIGURA DE MARIO BUSCHIAZZO ${ }^{1}$}

\author{
Mario José Buschiazzo (1902-1970) suele ser \\ reconocido en la literatura especializada por \\ su aporte al conocimiento de la historia de la \\ arquitectura argentina y latinoamericana, y \\ muy especialmente, de la arquitectura colonial² \\ (Schávelzon, 1988; De Paula, 1999; González, \\ 2004, Penhos, 2011; Gutiérrez, 2004; Alexan- \\ der, 1999).
}

Su trabajo como restaurador y, posteriormente, como investigador y motor de estudios relacionados con la historia de la arquitectura americana ha dejado una huella en el campo historiográfico. Buschiazzo trabajó en la Dirección General de Obras Públicas de la Nación y en el Ministerio de Obras Públicas de la Provincia de Buenos Aires, destacándose en su rol como asesor de la Comisión Nacional de Monumentos y Lugares Históricos, creada en 1940. Allí colaboró con la publicación del Boletín y cumplió el rol de restaurador de edificios. Paralelamente, en 1935, Buschiazzo fue nombrado Pro-

1 Este trabajo se enmarca dentro de la tesis de maestría de la autora, que estudia el interés de Buschiazzo por la arquitectura americana contemporánea a partir de los casos aquí analizados y otros, y busca entender de qué modo este interés iba de la mano de su ideología.

2 En este trabajo, se entenderá a lo colonial en arquitectura como "la actividad proyectual y edificadora llevada adelante durante el período de dominación hispánica" (González, 2004, p. 107) fesor Adjunto en la Escuela de Arquitectura, en lo que todavía era la Facultad de Ciencias Exactas, Físicas y Naturales de la Universidad de Buenos Aires (UBA). Por entonces escribió varios artículos y en 1940 publicó su primer libro, La arquitectura colonial en Hispanoamérica. De allí en más, Buschiazzo escribió una importante cantidad de libros y artículos relacionados con cuestiones de orden regional. ${ }^{3}$

En 1946 se creó, bajo la dirección de Buschiazzo, el Instituto de Arte Americano (IAA), dependiente de la UBA. EI Instituto tendría un enfoque "continental, pretendiendo abarcar en sus investigaciones y publicaciones todo el arte de la parte sur de América" (Buschiazzo, 1962, p. 319). Con tal fin, el IAA publica, desde 1948 y durante toda la gestión de Buschiazzo, los reconocidos Anales, cuyas páginas estarían abiertas a "todos los investigadores americanistas". Desde su lugar de director, Buschiazzo impulsó diversas actividades de gran importancia relacionadas con la historia de la arquitectura: exhibiciones, visitas de profesores extranjeros y publicaciones diversas. Buschiazzo dirigió el IAA hasta 1970, año de su fallecimiento.

3 Por un lado, publica varios volúmenes para la serie sobre Arquitectura Nacional de la Academia Nacional de Bellas Artes; por el otro, diversos libros independientes de los escritos para la Academia. Asimismo, colabora en la escritura de la Historia del Arte Hispano-Americano, dirigida por Diego Angulo Iñiguez y editada por Salvat. Escribe, también, varios artículos relacionados con la cuestión de Latinoamérica y con temas de orden nacional y que se publican en diversos medios: La Nación, La Prensa, Lasso, Estilo, La Razón (Schávelzon, 1988) 
Hoy en día, Buschiazzo es considerado un referente imprescindible a la hora de pensar la arquitectura colonial. Sin embargo, su trayectoria deja entrever un perfil que poco parece tener que ver con su interés por lo colonial: su relación con la arquitectura americana contemporánea. En 1955, siete años después del comienzo de la publicación de los reconocidos Anales (que en general se centraban en cuestiones relacionadas con "lo colonial”), el Instituto de Arte Americano e Investigaciones Estéticas (IAA) comenzó a publicar una serie llamada "Arquitectos Americanos Contemporáneos”. Esta serie tenía, según el propio Buschiazzo, la llamativa intención de "mantener en los trabajos un sentido de rigurosa actualidad, evitando el aspecto arqueológico que podría darle la investigación del pasado si no se la ligara con el presente" (Buschiazzo, 1962, p. 321-322). Más allá de este hecho en particular, la relación de Buschiazzo con la arquitectura americana contemporánea se construyó a partir de múltiples frentes que se relacionan, en gran parte, con la arquitectura de los Estados Unidos. ${ }^{4}$

A lo largo de este trabajo se estudiará la relación de Buschiazzo con la arquitectura americana contemporánea, principalmente entre los años 1955 (cuando se lanza la colección de Arquitectos Americanos Contemporáneos) y 1970 (año de su fallecimiento), con el objetivo de construir una imagen más rica y compleja del arquitecto que aquella obtenida únicamente a

4 Pocos autores han hecho mención de este interés de Buschiazzo por los Estados Unidos (Aliata, Ballent, 1990; Silvestri, 2004; Viñuales, 2015; Schávelzon, 2008) partir de sus estudios acerca de lo colonial, o de su trabajo como restaurador. En una primera sección, se analizará la relación de Buschiazzo con la arquitectura americana contemporánea, a través de la serie "Arquitectos Americanos Contemporáneos" y de su libro La arquitectura en la República Argentina: 1810-1930. En una segunda sección, en tanto, se estudiará su mirada frente a los Estados Unidos, a través de sus textos, sus viajes, sus vínculos y sus cartas. La hipótesis que guiará el trabajo es que, a diferencia de su imagen tradicional, hay en la figura de Mario Buschiazzo un interés evidente hacia la "arquitectura americana contemporánea".

\section{BUSCHIAZZO Y LA "ARQUITECTURA AMERICANA CONTEMPORANEA"}

\subsection{LA SERIE "ARQUITECTOS AMERICANOS CONTEMPORÁNEOS"}

EI IAA comienza a publicar, en 1955, una serie de libros bajo el titulo de "Arquitectos Americanos Contemporáneos". La decisión de Buschiazzo de publicar una serie como aquella, que reúne a arquitectos de toda América, incluyendo de los Estados Unidos, resulta al menos digna de atención en un momento en que Estados Unidos estaba construyendo la idea de una "arquitectura latinoamericana": en ese mismo año el MoMA presenta la exhibición “Latin American Architecture since 1945". Mientras la exposición del MoMA se ocupaba de mirar específicamente la arquitectura latinoamericana, los libros del IAA se ocupaban de lo americano en un sentido global. 
La serie comienza a publicarse tras una exposición del IAA sobre las obras del arquitecto Amancio Williams. En una carta enviada por Buschiazzo a Williams en octubre de 1955, el director del Instituto le hace saber al arquitecto -quien se encontraba en los Estados Unidos, invitado por el Departamento de Estado- que la exposición había sido un éxito, salvo por los catálogos, que a su gusto habían estado por debajo del nivel deseado. No obstante, Buschiazzo le comenta a Williams que el arquitecto Prebisch, interventor de la facultad, había quedado tan entusiasmado con la exposición que había llamado al contador de la facultad para imprimir un nuevo folleto o pequeño libro que contuviera las obras presentadas allí más un breve texto preliminar. El arquitecto Saal se ocuparía de seleccionar las láminas y Raúl González Capdevila de la redacción del texto.

El catálogo de la exposición se correspondió con la publicación número dos de los "Cuadernos del Instituto de Arte Americano", titulada "Cinco proyectos del Arq. Amancio Williams". En la presentación, titulada "Nuestra Contribución", Buschiazzo (1955) explica que era preciso, en ese determinado momento -tras la Revolución Libertadora-, que la obra de los arquitectos argentinos encuadrados "formalmente en las tendencias contemporáneas" fuera divulgada. Esto se debía a que "por la sinceridad con que han abrazado esa causa" podían constituir "un notable aporte para la formación" de los estudiantes. Buschiazzo explica que en este sentido, Amancio Williams era "uno de los más definidos representantes de las tendencias actuales". Y aunque sus obras habían traspuesto las fronteras, "lamentablemente" no eran tan conocidas en la Argentina.

Los arquitectos o estudios de arquitectura abordados por la serie "Arquitectos Americanos Contemporáneos" son: Amancio Williams, Eduardo Catalano, SOM, Lucio Costa, Paul Rudolph, Félix Candela, Bresciani-Valdéz-Castillo-Huidobro, Eladio Dieste, SEPRA, Mario Roberto Álvarez, Eero Saarinen y Philip Johnson.

Es notorio que no parece existir una coherencia general entre los diversos volúmenes de la serie en lo que a armado refiere. En general, cada autor compuso el libro sobre el arquitecto asignado a su gusto, y a partir de la organización que creyó más conveniente para el caso que le tocaba exponer. Así, algunos autores decidieron poner más énfasis en el análisis de obras, mientras otros buscaron situar a los arquitectos en contexto para así entender su rol en una determinada época y otros pretendieron, más bien, tratar de explicar la obra general de su arquitecto a partir de ciertas ideas o conceptos.

No obstante, parecería haber un cierto consenso entre los autores en cuanto a quiénes eran los personajes más destacados del mundo "contemporáneo" a nivel global. Hay ciertas referencias que se repiten en varios de los libros: Mies van der Rohe, Gropius, Frank Lloyd Wright y, por supuesto, Le Corbusier. 


\subsection{LA ARQUITECTURA EN LA REPÚBLICA ARGENTINA} 1810-1930

En el libro La arquitectura en la República Argentina 1810-1930, ${ }^{5}$ publicado en 1966, Buschiazzo intenta realizar un panorama sintético de lo que fue la historia de la arquitectura en nuestro país. Para ello, divide la historia en dos períodos: 1810-1862 y 1862-1930. Al centrarse en el segundo período, Buschiazzo critica la utilización de "extranjerismos" y del "eclecticismo historicista", definiendo a los estilos como un "mal inevitable" (p. 22) de los primeros años del siglo XX.

Buschiazzo explica que hacia el final de la Primera Guerra Mundial se dio un nuevo "baile de máscaras", por lo que se levantaron voces defensoras de lo americano, unificadas en lo que define la "restauración nacionalista" (en referencia directa a la expresión de Ricardo Rojas). Para Buschiazzo, “tal reacción se apoyaba sobre bases endebles, porque en definitivas cuentas también echaba mano del historicismo anacrónico, pero por lo menos tenía la disculpa de su patriótica aunque equivocada intención" (p. 29). En esta frase se expresa su posición frente a las ideas de Rojas y personajes como Guido y Noel. Buschiazzo considera que este

5 La colección privada de libros de Buschiazzo (que se encuentra en la Biblioteca "Prof. Leopoldo Herrera" de Resistencia) incluye libros de diversas áreas - no solo de arquitectura e historia del arte, sino que también cuenta con libros de historia general, de música y de filosofía, entre otros-, incluidos muchos tomos que tratan cuestiones vinculadas a la arquitectura contemporánea. Estos libros, con sus anotaciones y comentarios, prueban que Buschiazzo era un ávido lector, entre otras, de esta temática.

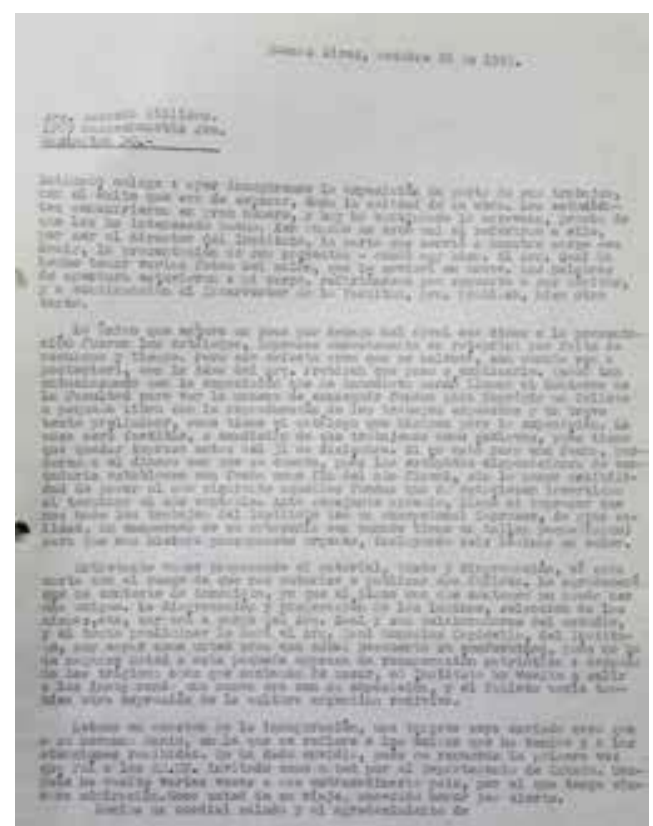

Figura 01.

Carta de Mario Buschiazzo a Amancio Williams, 26 de octubre de 1955. Disponible en el Archivo Buschiazzo del Instituto de Arte Americano e Investigaciones Estéticas “Mario J. Buschiazzo”. 
"movimiento" era negativo "por cuanto significaba pretender detener el curso del tiempo" y "estaba destinado a morir" (p. 30).

Pero "en medio de esta baraúnda de estilismos y de intentos fallidos", dice Buschiazzo, existieron quienes levantaron su voz "Ilamando a la cordura y tratando de encontrar la buena senda" (p. 30). Y destaca aquí a los "martinfierristas" entre los cuales se encontraba Alberto Prebisch, el "verdadero precursor de la arquitectura moderna en Argentina” (p. 30).

De todos modos, el acontecimiento que según Buschiazzo pudo haber sido trascendental en la arquitectura en la Argentina fue la visita de Le Corbusier en 1929. Buschiazzo no ahorra elogios a la hora de referirse a Le Corbusier: lo describe como un "genial arquitecto suizo" y califica al libro "Precisiones" como "magnífico". Pero dicho libro "no ha tenido ni tiene aquí la difusión que se merece" (p. 32). Y eso mismo sucede en general con sus ideas y con las de otros contemporáneos que reflexionaban sobre la arquitectura del momento:

las ideas y las conferencias de Le Corbusier no tuvieron la resonancia que merecían. Las publicaciones de la 'Bauhaus', de Weimar, y de 'L'Espirit Nouveau', los resultados del primer congreso del C.I.A.M. (Congrès Internationaux d'Architecture Moderne), solo eran conocidos por unos pocos, sin que trascendieran al grueso de los profesionales, todavía reacios e impermeables a las nuevas ideas (p. 32).
La explicación que encuentra Buschiazzo a esta falta de interés por la obra de arquitectos como Le Corbusier es que el país "no estaba aún maduro para ese avance, y el clima intelectual promedio seguía estancado en una rutina pasatista" (p. 32). A diferencia de lo que sucedió en Brasil, solo con el paso de los años fue tomando forma el "proceso renovador" en la Argentina. Pero aún así, la visita de Le Corbusier fue, para Buschiazzo, “un jalón fundamental en la evolución de nuestra arquitectura" porque marcó el fin de "un largo proceso de extravíos y anacronismos estilísticos y señaló la ruta a seguir en el futuro" (p. 32).

\section{BUSCHIAZZO Y LOS ESTADOS UNIDOS}

\subsection{LOS TEXTOS}

\subsection{1. "UN PRECURSOR AMERICANO DEL} FUNCIONALISMO"

El artículo "Un precursor Americano del Funcionalismo" (1935), es la segunda publicación dedicada a la arquitectura que se conoce de Buschiazzo. El protagonista del escrito es el arquitecto norteamericano Louis $\mathrm{H}$. Sullivan, una figura imprescindible del "movimiento inicial de la arquitectura contemporánea”, pero a quien no se suele nombrar cuando se mencionan a los precursores "del nuevo movimiento arquitectónico" (entre quienes sí suelen aparecer José María Olbrich, Theodor Fischer y Peter Berehns). Según Buschiazzo, esto es sumamente injusto y se debe a "la eterna miopía europea para toda visión americana” (p. 166), lo que resulta "tanto más lamentable desde nuestro punto de vista cuanto que se trata de un ame- 
ricano" (p. 166). Así, ya desde sus inicios Buschiazzo propone la idea de una América global.

No obstante, aclara, en los Estados Unidos sí se ha comenzado a valorar su aporte, "viendo en él al maestro de la nueva generación encabezada actualmente por su discípulo y continuador Frank Lloyd Wright, y asignándole el lugar de "primer americano modernista'” (p. 166). Buschiazzo especifica que esta idea tiene sentido si se comprende a la tendencia funcionalista como aquella "rigurosamente subordinada al principio de necesidad que origina y rige toda obra arquitectónica y cuya plástica trasciende sinceramente de la aplicación racional de sus elementos constructivos" (p. 166).

Haciendo referencia a la autobiografía de Sullivan, Buschiazzo explica que para apoyar su teoría de aquel como pionero, resulta imprescindible la "doctrina medular que rigió toda su obra", resumida en la frase "la función determina la forma; la forma expresa la función” (p. 166). Es esta, para Buschiazzo, la definición más exacta del "funcionalismo puro".

Según Buschiazzo, Sullivan fue pionero en "expresar y acusar la construcción esquelética en hierro aplicada a los rascacielos, que constituyen un programa utilitario y por ende específicamente funcional" (p. 167). Y en esto reside la clave de su funcionalismo: Sullivan fue el primero para quien la sustitución del muro portante por un sistema de vigas y columnas implicó “una nueva organización espacial”.

\subsubsection{DE LA CABAÑA AL RASCACIELOS}

Tras ser becado por la American Federation of Learning Societies, Buschiazzo publica, en 1945, el libro De la cabaña al rascacielos. En el mismo, busca explicar de qué modo fueron cambiando los modos de construir y las tipologías edilicias a lo largo de la historia de los Estados Unidos. Y es notorio que, dentro de la bibliografía que menciona, hace referencia al libro Sticks and Stones de Lewis Mumford (1924). A lo largo de todo el libro, es posible comprobar que Mumford fue un referente muy presente para Buschiazzo.

Buschiazzo divide al libro en 5 capítulos ordenados cronológicamente, partiendo de la época colonial y llegando hasta los primeros años del siglo XX. En el último capítulo, llamado "vértigo", Buschiazzo explica que tras la Primera Guerra Mundial el exceso de riqueza se tradujo en un desenfrenado impulso constructivo. Solo mientras se desarrollaba tan desmesurada producción lograron encauzarse las tendencias arquitectónicas hacia soluciones propias, sobre todo en el ámbito de la arquitectura privada: "en bancos, establecimientos industriales y especialmente en los rascacielos, los norteamericanos han llegado a crear una arquitectura propia, inconfundible, cuyo proceso evolutivo es singularmente interesante" (p. 74).

Es en este contexto que Buschiazzo inserta al rascacielos, "el más apasionante y norteamericano de los problemas arquitectónicos". Lo primero que hace el autor al referirse a los ras- 


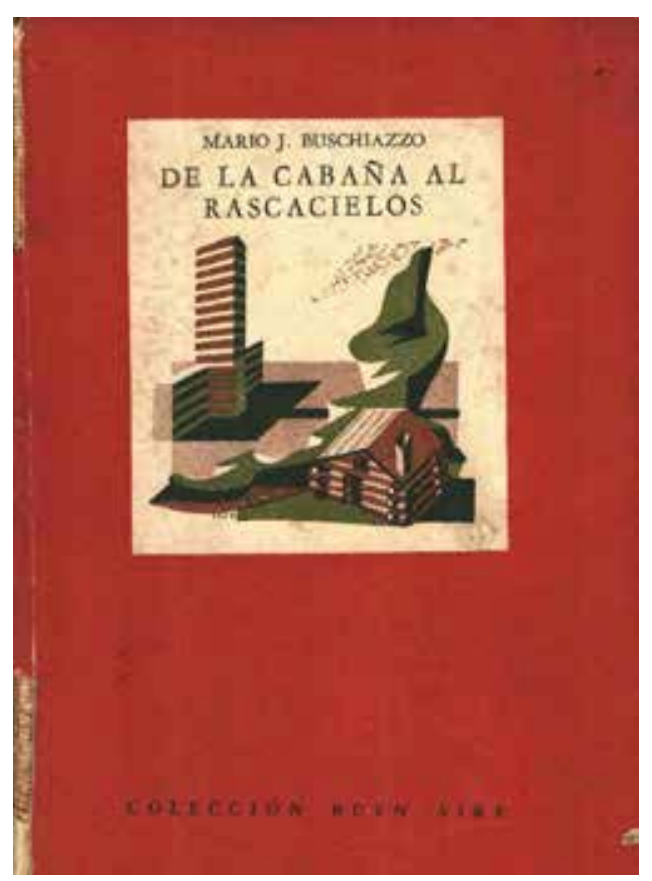

Figura 02

Tapa del libro De la cabaña al rascacielos, escrito por Mario Buschiazzo y publicado en 1945. cacielos es aclarar que no surgió en Manhattan por el "apeñuscamiento" urbano (la cuna del rascacielos es Chicago, según él) ni fue resultado de una "necesidad": "lo que ahora es la solución obligada de un pavoroso problema de congestión, fue al principio un simple alarde de técnica constructiva" (p. 76).

Según Buschiazzo, hasta 1881 los edificios no excedían los diez pisos por cuestiones técnicas. Pero una vez superadas estas cuestiones, comenzó la encrucijada estética: por un lado estaban los arquitectos como Sullivan, que remarcaban la verticalidad y dejaban de lado los órdenes clásicos sugiriendo nuevas formas ornamentales que no quitaran valor a los elementos verticales y por el otro, los arquitectos neoyorquinos, muchos de ellos egresados de la Escuela de Bellas Artes de París, quienes

no se atrevieron a romper con los cánones clásicos y cayeron en el absurdo de aplicar las columnas y pilastras de los templos romanos a un programa arquitectónico sustancialmente distinto...optaron por aplicar un orden con su correspondiente entablamento cada uno o dos pisos, cortando así la verticalidad del rascacielos(p. 80),

lo que para Buschiazzo resultaba en una "derrota estética evidente".

Pero más allá de la discusión estética, Buschiazzo asegura que tras la guerra en Europa, la lucha por ver quién llegaba más alto “alcanzó 
caracteres de morbosidad". Los rascacielos dejaron de levantarse por la necesidad de tener más oficinas: ahora se construían por el afán de record. Buschiazzo opina que son "el producto de una época de exacerbación" de un momento de absoluta prosperidad económica: "en la actualidad el promedio de edificios neoyorquinos oscila alrededor de los 35 pisos. Estos son los rascacielos 'auténticos', como expresión arquitectónica surgida de la necesidad (...) Pero no puede negarse que en su momento, los rascacielos monstruosos crearon un nuevo género de belleza en la que el tamaño juega un papel emocionante y decisivo" (p. 84).

Buschiazzo ubica a dos arquitectos estadounidenses, Sullivan y Saarinen como los precursores de lo que se estaba realizando en términos de rascacielos y asegura que su influencia llegó incluso hasta nuestro país: "actualmente no hay rascacielos que no siga la tendencia preconizada por Sullivan y lograda por Saarinen, incluso Buenos Aires, como lo prueba el edificio Kavanagh" (p. 82).

\subsubsection{SOM}

El libro dedicado al estudio estadounidense SOM, escrito por Mario Buschiazzo (1958) y número tres de la serie "Arquitectos Americanos Contemporáneos" comienza con una evidente revalorización de la arquitectura contemporánea de los Estados Unidos: “La arquitectura contemporánea de los Estados Unidos de Norte América ofrece el edificante ejemplo de la recuperación de sus valores tradicionales, des- pués de medio siglo de extravíos académicos de indudable procedencia europea" (p. 9). Estados Unidos es un ejemplo a seguir por haber logrado recuperar sus valores tradicionales en la arquitectura.

Tras la Primera Guerra Mundial, las voces europeas que reclamaban una arquitectura que se adaptara al momento, no lograron hacerse eco en Norteamérica frente a los preceptos academicistas. Tampoco lo lograron los "grandes maestros europeos" que Ilegaron a Estados Unidos desplazados de Europa: "durante muchos años Wright, Saarinen, Mies van der Rohe, Neutra o Gropius fueron vox clamantis in deserto, y sus realizaciones no trascendieron más allá del grupo de los iniciados, sin alcanzar a revolucionar la enorme masa de actividad constructiva norteamericana" (p. 9). Quienes seguían por los viejos cauces eran arquitectos "de indudable valía" como McKim Mead y White o Gilbert- "pero más proclives a tomar la senda de los éxitos fáciles que a afrontar una lucha de improbable y dudoso resultado" (p. 10). Para Buschiazzo lo "dramático" de esto es que los Estados Unidos habían sabido tener una arquitectura propia -como la de la época de los pioneros o la Escuela de Chicago- y “lamentablemente" la habían perdido. Centrándose en la Escuela de Chicago, Buschiazzo asegura que la misma no fue tal solo gracias a los adelantos técnicos: aquellos fueron "seguidos por una lógica interpretación de las posibilidades que brindaban, llegando a soluciones sencillas y claras" (p. 12). La Escuela de Chicago logró fusionar "armónicamente" arte e 
ingeniería. Y más allá de que hubieron ciertas "concesiones al pasatismo", aquellas quedaron relegadas ya que por encima de ellas "se podía leer con claridad la estructura metálica transformada en instrumento de expresión artística, superando así al estilismo histórico" (p. 14). De esta manera, asegura Buschiazzo, Chicago había conseguido una "auténtica arquitectura".

Pero mientras la arquitectura del oeste "maduraba", dice el autor, en el Este -sobre todo en Nueva York- el academicismo fue adquiriendo cada vez más fuerza. Y en esta lucha entre tendencias opuestas, la Exposición Internacional llevada a cabo en Chicago en 1893 terminó de definir el triunfo a favor de "la rama menos representativa de los auténticos valores universales" (p. 14). Tras esta experiencia se dio, para Buschiazzo, un "derrumbe arquitectónico" que se extendió por todo el país, llenándolo de "palacetes-castillos, casas neogriegas, capitolios corintios, bancos dóricos y universidades góticas" (p. 16). La claridad de expresión de la Escuela de Chicago fue reemplazada por una búsqueda formalista, y solo algunos casos aislados -como los de Frank Lloyd Wright, Richard Neutra, Mies van der Rohe y Gropius- se dejaron oír cada tanto.

Este momento de la arquitectura norteamericana, definido por Buschiazzo como "caótico", se extendió hasta el primer cuarto del siglo XX. Ya era inadmisible que en los Estados Unidos se siguiera dando tal incongruencia entre sus expresiones artísticas y su realidad económica, social y política. Poco a poco, arquitectos norteamericanos se fueron uniendo a los "maestros europeos", plegándose al "movimiento moderno". Mientras tanto, el Museo de Arte Moderno de Nueva York fue, según el autor, esencial para concientizar al ambiente artístico, probando que "el país estaba ya maduro (...) para comprender el sentido de la arquitectura actual” (p. 17).

Buschiazzo considera que la arquitectura "actual" estadounidense ha logrado "traducir el triunfo de la técnica y la potencialidad económica, las dos características más notorias del pueblo norteamericano" (p. 18). Y esto ha sido plenamente realizado en la obra de Skidmore, Owings y Merril.

\subsection{LOS VIAJES Y VÍNCULOS}

Buschiazzo realizó diversos viajes a Norteamérica. En 1941 fue becado por la American Federation of Learning Societies y el Departamento de Estado de los Estados Unidos de América para una "misión de intercambio cultural y conferencia” (AAVV, 1999, p. 48); en 1960 obtuvo la Beca Rockefeller que le permitiría estudiar en las Universidades de Ann Arbor, Michigan y Austin. Asimismo, en 1950 fue Invitado de Honor de la Bilbioteca del Congreso de Washington, lo que le permitió asistir al "International Colloqium on Luso-Brazilian Studies"; En 1954 fue Invitado de Honor al Metropolitan Museum of Art de Nueva York, asistiendo al Congreso Internacional de Historia del Arte; y en 1961 fue invitado y organizó la sección de Arte Hispano-Americano del XX Congreso Internacional de 
Historia del Arte realizado en Nueva York. Asimismo, Buschiazzo aprovechaba invitaciones a actividades académicas en diversos países para pasar, a su vuelta, por los Estados Unidos.

Con respecto a sus relaciones institucionales, como director del IAA, Buschiazzo mantuvo contacto con distintas instituciones estadounidenses como por ejemplo la New York Public Library, la University of California, Columbia University, Harvard University, New York University y la secretaría general de la Organización de Estados Americanos, entre otras. Y no solo se enviaba correspondencia con las mismas sino que visitó a varias de ellas en sus viajes.

Un último eje importante eran sus relaciones académicas y personales. Buschiazzo tenía contacto con diversos personajes que se encontraban trabajando en los Estados Unidos como ser Kenneth Conant, Harold E. Wethey, Martin Soria, Robert C. Smith y Pál Kelemen. Asimismo, Raquel Buschiazzo cuenta que Mario y su mujer, María Ester García Martínez, eran muy amigos de Dudley Easby -quien fuera secretario del Metropolitan Museum of Art de Nueva York- y su mujer. También era amigo de Lester Walker, quien daba clases en la Universidad de Georgia. Raquel narra que su abuelo estuvo un mes ayudando a Walker a preparar las clases sobre arte y arquitectura americanos y que dio la clase inaugural sobre el tema (R. Buschiazzo, entrevista personal, 10 de abril de 2016).

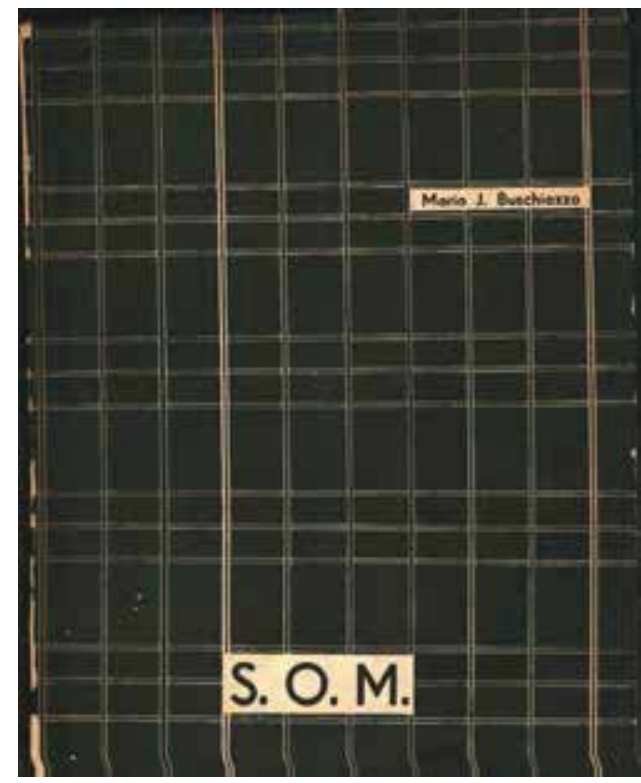

Figura 03

Tapa del libro SOM, de la serie "Arquitectos Americanos Contemporáneos", escrito por Mario Buschiazzo y publicado en 1958. 


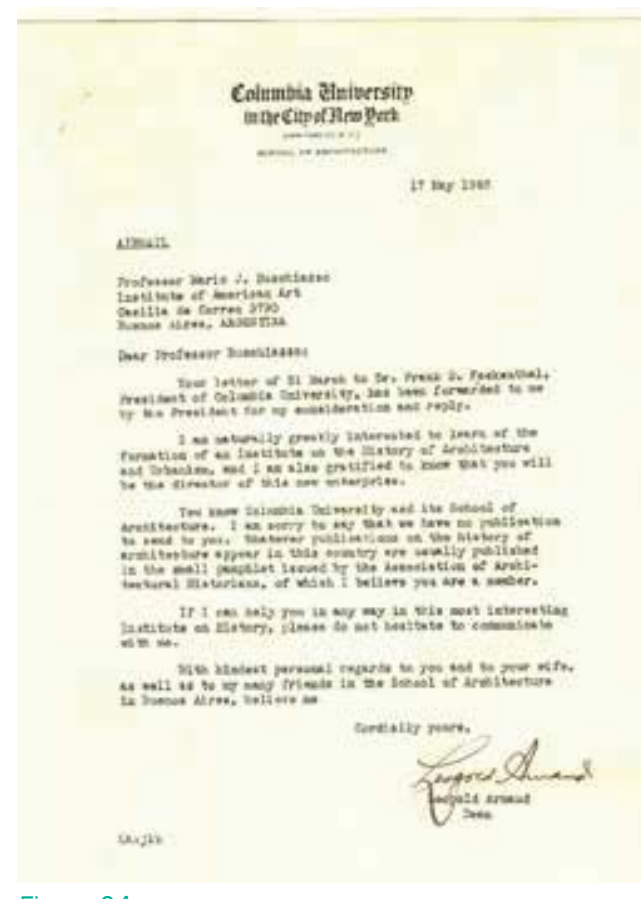

Figura 04.

Carta enviada por el Decano de la Escuela de Arquitectura de Columbia University, Leopold Arnaud, a Mario Buschiazzo el 17 de mayo de 1948.

Disponible en el Archivo Buschiazzo del Instituto de Arte Americano e Investigaciones Estéticas "Mario J. Buschiazzo".

\subsection{LAS CARTAS}

Como se ha mencionado anteriormente, en ocasión de la exposición del IAA sobre los proyectos de Amancio Williams, Buschiazzo le escribe una carta (1955a) a su colega -quien estaba en los Estados Unidos- pidiendo autorización para publicar un libro sobre dichos proyectos:

Leímos en ocasión de la inauguración, una tarjeta suya (...) en la que se refiere a los éxitos que ha tenido y a las atenciones recibidas. Me ha dado envidia, pues me recuerda la primera vez que fui a los EE.UU. invitado como usted por el departamento de Estado. Después he vuelto varias veces a ese extraordinario país, por el que tengo sincera admiración.

Sin ir más lejos, Buschiazzo admite a Williams su admiración por los Estados Unidos y da cuenta de sus varias visitas a aquel país.

Buschiazzo parece admirar de los Estados Unidos, entre otras cosas, su orden, su rigurosidad. Así, en una carta que envía a Diego Angulo Iñiguez (1953), justifica haberse quejado de la falta de severidad en los investigadores españoles y asegura:

Mi carta quejándome de la informalidad e impuntualidad de los investigadores españoles ha levantado un sin fin de protestas, pero me alegro de haberlo hecho (...) Mal que les pese, hace falta un poco de organización yanqui. 
Por otro lado, Buschiazzo hizo un gran esfuerzo para lograr que un flujo constante de libros entrara al IAA por medio del intercambio. Y logró armar una amplia red. Muchas de las instituciones con las que se realizaban estos intercambios eran estadounidenses. Por ejemplo, con este objetivo de intercambio, Buschiazzo escribe una carta a Isabelle Entrikin de la Biblioteca Lincoln (1955b). En la misma, narra las diversas actividades que lleva a cabo el IAA y da como ejemplo una exposición realizada sobre la "Arquitectura Moderna en los Estados Unidos” en la cual se contó “con material facilitado gentilmente por el Sr. Allard, del servicio informativo de la Embajada" ${ }^{\text {. }}$. En la carta, parecería que Buschiazzo intentar dar al Instituto un carácter de seriedad por medio de sus contactos con instituciones o personajes estadounidenses. Así, explica que "el Instituto publica obras de arte, y edita sus Anales, con colaboraciones de profesores de universidades de toda América", aclarando que entre sus colaboradores "figuran los profesores Kenneth Conant, de la Universidad de Harvard, Robert C. Smith, de la Univ. de Pennsylvania, Harold E. Wethey, de la Universidad de Michigan, Martin S. Soria, del Michigan State College, etc.". Incluso, hace explícita esta noción de que la formalidad del IAA

6 La Biblioteca Lincoln se encontraba en Buenos Aires pero formaba parte de una iniciativa por medio de la cual bibliotecas estadounidenses eran instaladas en distintos países con el fin de "dar a los extranjeros acceso a impresiones americanas básicas y otros materiales" en un momento en que el apoyo de Latinoamérica resultaba vital para los Estados Unidos. Isabelle Entrikin, la bibliotecaria estadounidense con la que se escribe Buschiazzo, "era muy capaz y popular en la Argentina” (Murphy, 1990)

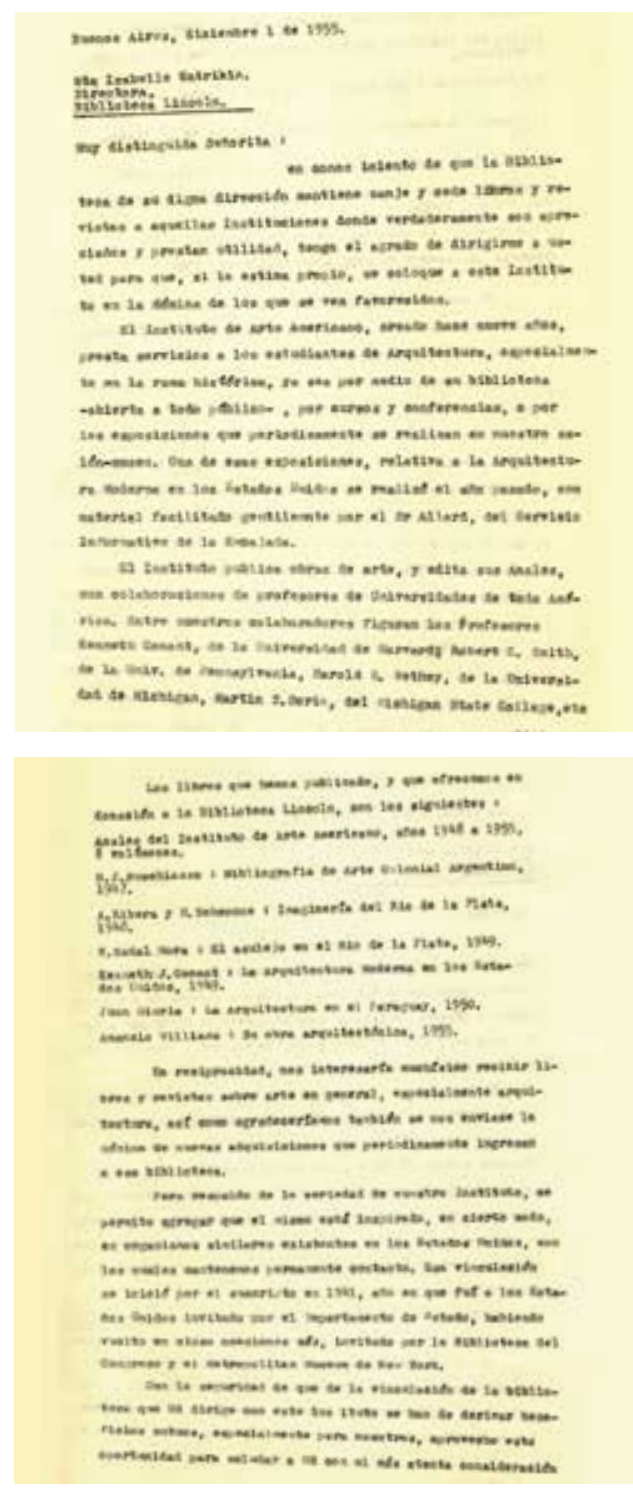

Figuras 05. y 06.

Carta enviada por Mario Buschiazzo a la Directora de la Biblioteca Lincoln, Isabelle Entrikin, el 1 de diciembre de 1955. Disponible en el Archivo Buschiazzo del Instituto de Arte Americano e Investigaciones Estéticas “Mario J. Buschiazzo”. 
va de la mano de haber tomado como modelo otros institutos norteamericanos, de la relación que mantiene con ellos y de sus propias visitas como invitado a los Estados Unidos:

para respaldo de la seriedad de nuestro instituto, me permito agregar que el mismo está inspirado, en cierto modo, en organismos similares existentes en los Estados Unidos, con los cuales mantenemos permanente contacto. Esa vinculación se inició por el suscripto en 1941, año en que fui a los Estados Unidos invitado por el Departamento de Estado, habiendo vuelto en cinco ocasiones más, invitado por la Biblioteca del Congreso y el Metropolitan Museum de Nueva York.

Esta carta permite ver de modo claro, cuál era la postura de Buschiazzo frente al país norteamericano, y deja entrever un cierto dejo de admiración por el mismo y de orgullo por las relaciones que ha sabido lograr con sus personajes e instituciones.

\section{CONCLUSIONES}

A partir de todo lo estudiado, es evidente que Mario Buschiazzo tenía un interés por la "arquitectura americana contemporánea” y que el mismo iba fuertemente ligado a su atracción por los Estados Unidos. Su primer texto, con el cual comenzó a adentrarse en la historia de la arquitectura ("Panorama histórico de los Estados Unidos a través de su Arquitectura"), hacía foco en la arquitectura norteamericana. Y luego, no solo fue escribiendo sobre arquitectura contemporánea y norteamericana, sino que también se ocupó, como director del IAA, de realizar exposiciones y editar libros que tuvieran como eje la arquitectura de los Estados Unidos o bien la arquitectura contemporánea en América.

En primer lugar, el aporte que realizó Buschiazzo con la edición de la serie "Arquitectos Americanos Contemporáneos" resultó de particular interés en el momento en que se publicó, al brindar un panorama de lo que se estaba realizando, arquitectónicamente, a nivel continental. La mirada americana en sentido amplio que propuso Buschiazzo en esta serie, como se ha visto, también es destacable si se tiene en cuenta que casi al mismo tiempo, se estaba fomentando la idea de una "América Latina" -lo que conlleva a un inevitable contraste con una "América Sajona” (Ardao, 1993)- por una institución como la del MoMA de Nueva York. Mientras los Estados Unidos buscaban acercarse a "Latinoamérica" mediante medidas que seguían remarcando las diferencias entre las Américas, Buschiazzo miraba a América como un todo.

En segundo lugar, a partir del libro La arquitectura en la República Argentina 1810-1930, resulta evidente que, a diferencia de otros personajes que integraban el campo de la historia de la arquitectura en la Argentina -como por ejemplo Martín Noel- Buschiazzo veía a lo colonial como algo a estudiar científicamente por ser parte de nuestra historia; no para ser replicado en el presente. Para él, las bases de la "restauración 
nacionalista" eran endebles porque se apoyaban en un "historicismo anacrónico". Y en paralelo, opinaba que la visita de Le Corbusier podría haber sido trascendental para la arquitectura argentina pero que el país no estaba intelectualmente preparado aún para comprenderlo.

Buschiazzo, entonces, no solo se diferenciaba de personajes como Noel a partir de su postura respecto de cómo hacer historia (Gutiérrez, 1992), sino también respecto de cuál era la arquitectura adecuada para el presente. Y el país que para Buschiazzo sí logró, tras años de desvaríos, encontrar su arquitectura propia, actual, era Estados Unidos. Tanto "Un precursor americano del funcionalismo" como De la cabaña al rascacielos como SOM hacen foco, desde distintos ángulos, en la arquitectura estadounidense. $Y$ en los tres casos, ya sea desde un arquitecto precursor, o una tipología, o la capacidad de plasmar las características de un pueblo en arquitectura, los Estados Unidos eran el ejemplo. Esto se ve, también, en las cartas institucionales que se han estudiado a lo largo de este trabajo.

Todo esto permite pensar a Buschiazzo desde una perspectiva más amplia, compleja y densa: Buschiazzo no fue únicamente un restaurador e historiador preocupado por lo colonial, sino que sus inquietudes se condensaron también en otras aristas, entre las que se encuentran lo contemporáneo y lo norteamericano.

RECIBIDO: 30 de junio 2016 ACEPTADO: 15 de noviembre 2016 


\section{BIBLIOGRAFIA}

Alexander, R.J. (1996-1997). Mario J. Buschiazzo: la audacia de un compromiso con América. Anales del Instituto de Arte Americano e Investigaciones Estéticas "Mario J. Buschiazzo", (31-32), 79-89.

Aliata, F. \& Ballent, A. (1990). Crítica e Historia: dos modelos alternativos frente a la arquitectura contemporánea. En Segundas Jornadas del Comité Argentino del Comité Internacional de Ciencias Históricas, Historiografía Argentina (1958-1988): una evaluación crítica de la producción histórica argentina, Paraná, 1988. (pp.186-196). Buenos Aires: Comité Argentino de Ciencias Históricas.

Ardao, A. (1980). Génesis de la idea y el nombre de América Latina. En A. Ardao (1993). América Latina y la latinidad. México: UNAM.

Buschiazzo, M. J. (1962). El Instituto de Arte Americano de la Facultad de Arquitectura y Urbanismo. Revista de la Universidad de Buenos Aires, 7(2), 317-322.

Buschiazzo, M. J. (1955). Nuestra contribución. Cinco proyectos del Arq. Amancio Williams, Cuadernos del Instituto de Arte Americano, (2), 4.

Buschiazzo, M.J. (1948). Presentación. Cánones de crítica, Cuadernos del Instituto de Arte Americano, (1), 1-3.

Buschiazzo, M.J. (1935). Un precursor americano del funcionalismo. Revista de Arquitectura, (172).
Buschiazzo, M.J. (1945). De la cabaña al rascacielos. Buenos Aires: Emecé.

Buschiazzo, M.J. (1971). La Arquitectura en la República Argentina, 1810-1930. Buenos Aires: Mac Gaul.

Buschiazzo, M.J. (1958). S.O.M. Skidmore. Owings y Merril. Buenos Aires: Instituto de Arte Americano e Investigaciones Estéticas.

De Paula, A. (1996-1997). Mario J. Buschiazzo y el Instituto de Arte Americano. Anales del Instituto de Arte Americano e Investigaciones Estéticas “Mario J. Buschiazzo, (31-32), 15-54.

De Paula, A. (Dir.) (1996-1999). Anales del Instituto de Arte Americano e Investigaciones Estéticas "Mario J. Buschiazzo", (31-32), $362 \mathrm{p}$.

González Montaner, B. (2004). Voz: "Colonial (Arquitectura)”. En F. Aliata; J.F. Liernur (Comps.). Diccionario de Arquitectura en la Argentina: estilos, obras, biografías, instituciones, ciudades. Buenos Aires: Agea.

Gutiérrez, R. (2004). Notas sobre la historiografía del arte y la arquitectura colonial americana. En R. Gutiérrez (Dir.) Historiografía Iberoamericana. Arte y Arquitectura (XVI-XVIII). Buenos Aires: Cedodal.

Gutiérrez, R. (1992). Origen historiográfico de la polémica Noel-Buschiazzo (1948-1950). Documentos de Arquitectura Nacional y Americana (DANA), (31/32), 11-14. 
Mumford, L. (1924). Sticks and Stones: A study of American architecture and civilization. New York: Boni and Liveright.

Murphy, E. (1990, January 30). Interview with Edmund Murphy. (A. Hansen, Interviewer). Recuperado de http://www.adst.org/OH\%20 TOCs/Murphy,\%20Edumund.pdf

Penhos, M. (2011). De categorías y otras vías de explicación: una lectura historiográfica de los Anales de Buenos Aires (1948-1971). En Memoria del III Encuentro Internacional sobre Barroco, Manierismo y transición al Barroco, 2011. Pp. 167-174. Pamplona: Fundación Visión Cultural.

Schávelzon, D. (1988). Bio-bibliografía de Mario Buschiazzo. Revista de la Sociedad Central de Arquitectos, (141), 24-29. Recuperado de http://www.iaa.fadu.uba.ar/?page_id $=57$

Schávelzon, D. (2008). Mejor olvidar: la conservación del patrimonio cultural argentino. Buenos Aires: Academia de Historia de la Ciudad de Buenos Aires; De los Cuatro Vientos Editorial.

Silvestri, G. (2004). Voz: "Historiografía”. En F. Aliata; J.F. Liernur (Comps.). Diccionario de Arquitectura en la Argentina: estilos, obras, biografías, instituciones, ciudades. Buenos Aires: Agea.

Viñuales, G.M. (2015). Mario José Buschiazzo y su visión americanista. En R. Gutiérrez; W. Rincón García; F. Vela Cossío (Coords.) Una empresa memorable de España hacia Amé- rica. La edición de Angulo Íñiguez, Marco Dorta y Buschiazzo sobre el arte americano (1945-1956). pp.79-86. Madrid: Rueda.

\section{CARTAS}

Carta de Mario Buschiazzo a Amancio Williams (enviada a Washington DC) el 26 de octubre de 1955a. Disponible en el Archivo Buschiazzo del Instituto de Arte Americano e Investigaciones Estéticas “Mario J. Buschiazzo”.

Carta enviada por Mario Buschiazzo a la Directora de la Biblioteca Lincoln, Isabelle Entrikin, el 1 de diciembre de 1955b. Disponible en el Archivo Buschiazzo del Instituto de Arte Americano e Investigaciones Estéticas "Mario J. Buschiazzo". 